\title{
Fractura de cadera por fragilidad en México: ¿En dónde estamos hoy? ¿Hacia dónde queremos ir?
}

\author{
Viveros-García JC,* Torres-Gutiérrez JL, ${ }^{* *}$ Alarcón-Alarcón T,*** Condorhuamán-Alvarado PY,*** \\ Sánchez-Rábago CJ,**** Gil-Garay E,**** González-Montalvo JI*****
}

Hospital Regional ISSSTE, León, Guanajuato, México

RESUMEN. Introducción: La fractura de cadera (FC) se considera la consecuencia más grave de las caídas y la osteoporosis. Dieciocho por ciento de las mujeres sufrirán una FC y $\mathbf{2 5 \%}$ muere en el primer año. Sólo $73 \%$ de los supervivientes caminará como lo hacía previamente. Conocemos poco de la asistencia y evolución de la FC en México. Objetivo: Revisar el estado actual de la literatura mexicana sobre $\mathrm{FC}$, comparar estudios mexicanos entre sí y con los indicadores de calidad. Metodología: Se realizó una búsqueda de artículos mexicanos publicados entre 2000 y 2017 con las palabras clave: fractura de cadera, ancianos, México y hip fracture en las bases de datos PubMed, EBSCO y Bibliomed. Resultados: Se incluyeron 22 artículos. No se encontraron ensayos clínicos, informes de unidades multidisciplinarias, registros, ni metaanálisis. La media de edad fue de 76.9 años y $67.2 \%$ fueron mujeres. No se encontraron trabajos que reportaran indicadores de calidad. La comorbilidad se describe poco. Las complicaciones más frecuentes de la $\mathrm{FC}$ fueron delirium, neumonía y úlceras por presión. La mortalidad en la fase aguda fue de 0.97 a $12.5 \%$. No se reportaron unidades ortogeriátricas. El costo de atención osciló entre 1,261 y 13,641 dólares estadounidenses (USD). Conclu-
ABSTRACT. Introduction: Hip fracture is considered the most serious consequence of falls and osteoporosis. $18 \%$ of women will suffer one and $25 \%$ die in the first year. Only $73 \%$ of survivors will walk as they did previously. We know little about the assistance and evolution of it in Mexico. Our goal was to review the current state of Mexican literature on hip fracture, compare Mexican studies with each other and with quality indicators. Methods: We conducted a search of Mexican articles published between 2000-2017 with the key words: hip fracture, elderly, Mexico and hip fracture (in English) in the databases PubMed, EBSCO and Bibliomed. Results: Twentyone articles were included. No clinical trials, multidisciplinary unit reports, records, or metaanalyses were found. The average age was 76.9 years and $67.2 \%$ were women. No papers were found to report quality indicators. Comorbidity is reported little. The most common complications of hip fractures were delirium, pneumonia and pressure ulcers. Mortality in the acute phase was 0.97 to $12.5 \%$. Special units were not reported. The cost of care oscillated between 1,261 and 13,641 USD. Conclusions: The scientific information on hip fractures in Mexico is sparse, heterogeneous

Nivel de evidencia: IV

\footnotetext{
* Servicio de Geriatría, Hospital Regional ISSSTE, León, Guanajuato, México. Geriatra en la Escuela de Fisioterapia, Escuela Nacional de Estudios Superiores Unidad León, UNAM, México.

** Jefe de Servicio de Geriatría, Hospital Regional ISSSTE, León, Guanajuato, México.

*** Unidad de Ortogeriatría. Servicio de Geriatría. IdiPAZ. Hospital Universitario La Paz. Madrid, España.

**** Traumatólogo en la Escuela de Fisioterapia, Escuela Nacional de Estudios Superiores Unidad León, UNAM, México.

***** Jefatura de Traumatología y de Geriatría, IdiPAZ. Hospital Universitario La Paz. Madrid, España.
}

Dirección para correspondencia:

JC Viveros-García

Av. Pradera Núm. 1101, Col. Aztecas, CP 37520, León, Guanajuato, México.

E-mail: drviveros.geriatria@gmail.com

Este artículo puede ser consultado en versión completa en http://www.medigraphic.com/actaortopedica 
siones: La información científica sobre FC en México es escasa, heterogénea y no permite obtener resultados concluyentes. Se requiere aumentar la cantidad y la calidad de la investigación en FC en México. Sería también conveniente difundir la utilidad de los equipos multidisciplinarios y registros de FC, lo que contribuiría a mejorar la atención.

Palabras clave: Fractura de cadera, ancianos, México, tratamiento, investigación.

\section{Introducción}

Los cambios demográficos de las últimas décadas han llevado a un incremento del número de adultos mayores. ${ }^{1}$ Este aumento se ha acompañado de mayor prevalencia de enfermedades crónicas no transmisibles como la osteoporosis (OP) y sus consecuencias, incluyendo las fracturas de cadera $(\mathrm{FC}){ }^{2}$

En México, una de cada cuatro personas adultas cursa con osteopenia u osteoporosis ${ }^{3}$ y se calculó a finales del siglo pasado un promedio de $100 \mathrm{FC}$ por día. ${ }^{4} \mathrm{Se}$ estima que entre 8.5 y $18 \%$ de las mujeres ${ }^{2,5}$ tendrán una FC a lo largo de su vida. La incidencia de FC en la Ciudad de México es de 1,725 casos en mujeres y 1,297 hombres por cada 100,000 habitantes, con una proyección de incremento hasta de siete veces para el año 2050 y en el año 2005 se reportaron 29,373 FC en México, ${ }^{6}$ mientras que en países como España tienen 41,000 FC anuales. ${ }^{7}$

Se considera que las FC son la consecuencia más grave tanto de las caídas como de la OP debido a su impacto funcional y su alta morbimortalidad. $8,9,10,11,12$ Tan sólo $73.6 \%$ de los pacientes recupera a los dos años una movilidad similar a la que tenía previo a la fractura. ${ }^{13} \mathrm{La}$ mortalidad en la fase aguda tras una FC suele ser inferior a $10 \% ;{ }^{9,14,15}$ sin embargo, después del primer año, entre $23.6 \%$ y $34.5 \%$ de las personas han fallecido. ${ }^{7,16,17}$ Otro punto que vuelve a la FC catastrófica es el costo asociado. En el año 2002 el Instituto Mexicano del Seguro Social (IMSS) gastó cerca de 1,927,072 dólares estadounidenses (USD) en atención a la FC. ${ }^{10}$ En México, el costo en la atención de una FC va de 5,803 a 11,800 USD, ${ }^{11}$ mientras que en un hospital público en España supone un gasto de 12,302 USD por evento. ${ }^{17}$

$\mathrm{El}$ paciente que sufre una FC suele ser muy complejo y presenta una gran heterogeneidad en sus condiciones clínicas, funcionales, cognitivas y sociales. Sin embargo, el prototipo es una mujer mayor de 80 años con enfermedades crónicas múltiples y un riesgo anestésico elevado. ${ }^{12}$ Por todo lo anterior, en los últimos años se han realizado múltiples esfuerzos para estudiar la FC e intentar mejorar su atención y pronóstico, tanto funcional como de vida, además de disminuir los costos hospitalarios. Esto a través de guías de and does not allow for conclusive results. Increasing the amount and quality of research in hip fractures in Mexico is required. It would also be advisable to disseminate the usefulness of multidisciplinary teams and registry of hip fractures, which would help to improve attention.

Key words: Hip fracture, elderly, Mexico, treatment, research.

práctica clínica, implementación de equipos multidisciplinarios, inclusión del geriatra en la atención desde la fase aguda y la formación de unidades de ortogeriatría, , $, 14,18,19$ además de la publicación de recomendaciones e indicadores de calidad en la atención. ${ }^{20}$ Todo ha permitido a países como España, entre muchos otros, disminuir los costos de atención, reduciendo fundamentalmente la estancia hospitalaria. $^{13,17}$ Sin embargo, la morbimortalidad de la FC sigue siendo considerablemente alta y se ha mantenido estable en los últimos años. ${ }^{21}$

En comparación con países desarrollados, México se encuentra todavía en cierta medida rezagado. No se cuenta aún con un registro nacional y los esfuerzos para mejorar la atención e investigación en FC siguen siendo aislados. Por otra parte, el número de unidades ortogeriátricas es insuficiente y los resultados asistenciales son sumamente heterogéneos, la evidencia en la literatura sobre FC es escasa y no se ha revisado ni puesto al día. ${ }^{15,22,23,24,25,26}$ Nuestro objetivo fue conocer el estado actual de la literatura científica mexicana en cuanto a la atención de la FC, comparar los resultados de equipos de investigación mexicanos y conocer el estado asistencial en cuanto a indicadores de calidad.

\section{Metodología}

Se realizó una búsqueda en PubMed, EBSCO y Bibliomedia usando las palabras clave: hip fracture, aged, México, elder, fractura de cadera y ancianos. Se incluyeron trabajos mexicanos de investigación publicados desde el año 2000 hasta 2017, en inglés y español. Se recopilaron los resultados de las variables demográficas y los tipos de fractura, los resultados postoperatorios incluyendo la mortalidad, complicaciones, marcha y funcionalidad; además de los costos de atención e indicadores de calidad basados en las guías $\mathrm{NICE}^{20}$ y las recomendaciones Best Practice Tariff. ${ }^{27}$ Se excluyeron artículos de revisión, reporte de casos y trabajos con fracturas fuera de la fase aguda. Se calculó el equivalente del costo de atención en dólares estadounidenses, ajustado a la fecha de publicación del artículo a través de la herramienta en línea FX-TOP. ${ }^{28}$ 


\section{Resultados}

Se encontró un total de 45 artículos, de éstos se eliminaron 23 trabajos y se incluyeron 22 (Figura 1). Dieciocho trabajos fueron sobre investigación clínica, nueve fueron prospectivos y nueve retrospectivos. ${ }^{11,15,23,24,29,30,31,32,33,34} \mathrm{El}$ resto fueron estudios sobre incidencia y costos..$^{5,6,35,36}$ No se encontraron registros regionales ni nacionales, ensayos clínicos aleatorizados o metaanálisis.

La mayoría de los trabajos se realizaron en la Ciudad de México en centros hospitalarios de traumatología y sus objetivos correspondían a características enfocadas a dicha especialidad. La media de pacientes incluidos en los estudios fue de 162 pacientes, con un rango de 18 a 1,000. La edad promedio en estos trabajos fue de 76.9 años y $67.2 \%$ correspondieron al género femenino (Tabla 1). Ninguno de ellos se realizó en unidades ortogeriátricas ni contó con un geriatra como autor.

Se observó que la comorbilidad es pocas veces reportada y cuando se hace, los resultados son muy heterogéneos, como consecuencia también lo es el riesgo quirúrgico ( $\mathrm{Ta}$ bla 2). No se encontraron trabajos que incluyeran índices de comorbilidad como el de Charlson o alguno similar. Únicamente tres trabajos consideraron el deterioro cognitivo como variable a incluir. No se encontraron reportes sobre índices de funcionalidad y características de la marcha previos a la fractura ni trabajos sobre características sociales que hayan demostrado ser importantes en el pronóstico, por ejemplo, si procedían de residencia o de su domicilio.

En cuanto a los tipos de fractura y su tratamiento quirúrgico (Tabla 3), cinco estudios incluyeron exclusivamente fracturas pertrocantéreas y uno, fracturas intracapsulares. Sólo un trabajo incluyó los tres principales tipos de fracturas, con una frecuencia de 36\% intracapsulares, 56\% pertrocantéreas y $8 \%$ subtrocantéreas. En lo que respecta al trata- miento instaurado, la hemiartroplastía y el DHS (Dinamic Hip Screw, DePuy/Synthes, Johnson \& Johnson) fueron los dispositivos más frecuentemente empleados. En un solo trabajo utilizaron la artroplastía total de cadera.

Los resultados asistenciales tras el tratamiento quirúrgico (Tabla 4) muestran que la complicación descrita más frecuente es el delirium; sin embargo, sólo se reportó en dos trabajos, seguida de la aparición de úlceras por presión que se registró también en dos estudios. En cuanto a la mortalidad, a los 30 días hubo una variabilidad importante de 0.97 a $12.5 \%$. Ningún trabajo evaluó el tiempo de espera entre el ingreso y la cirugía. En cuanto a la marcha, las definiciones operativas son poco claras y no se describen las características de ésta previo a la fractura (independiente o asistida, auxiliares de la marcha).

Por último, sólo tres trabajos analizaron los costos (Tabla 5) con una variabilidad importante. Tras convertir a dólares estadounidenses, ajustado a la inflación, los costos van de 1,261 a 13,641 USD, dependiendo del sistema mexicano de salud que atienda al paciente.

\section{Discusión}

En nuestra revisión encontramos que el número de publicaciones sobre FC en ancianos en México es escaso, con objetivos, resultados, metodología sumamente variables, así como calidad desigual.

La edad media de los pacientes fue de 76 años, lo que contrasta con países desarrollados como Australia y Nueva Zelanda donde la media es de 83 años ${ }^{19}$ o en España donde la media es de 86.2 años. ${ }^{17}$ Esto debido a la actual transición demográfica que se está desarrollando en México y que en países desarrollados tuvo lugar anteriormente. Al igual que en otros trabajos internacionales, la mayoría de pacientes con FC en México fueron mujeres (66.9\%). En España, Alarcón y

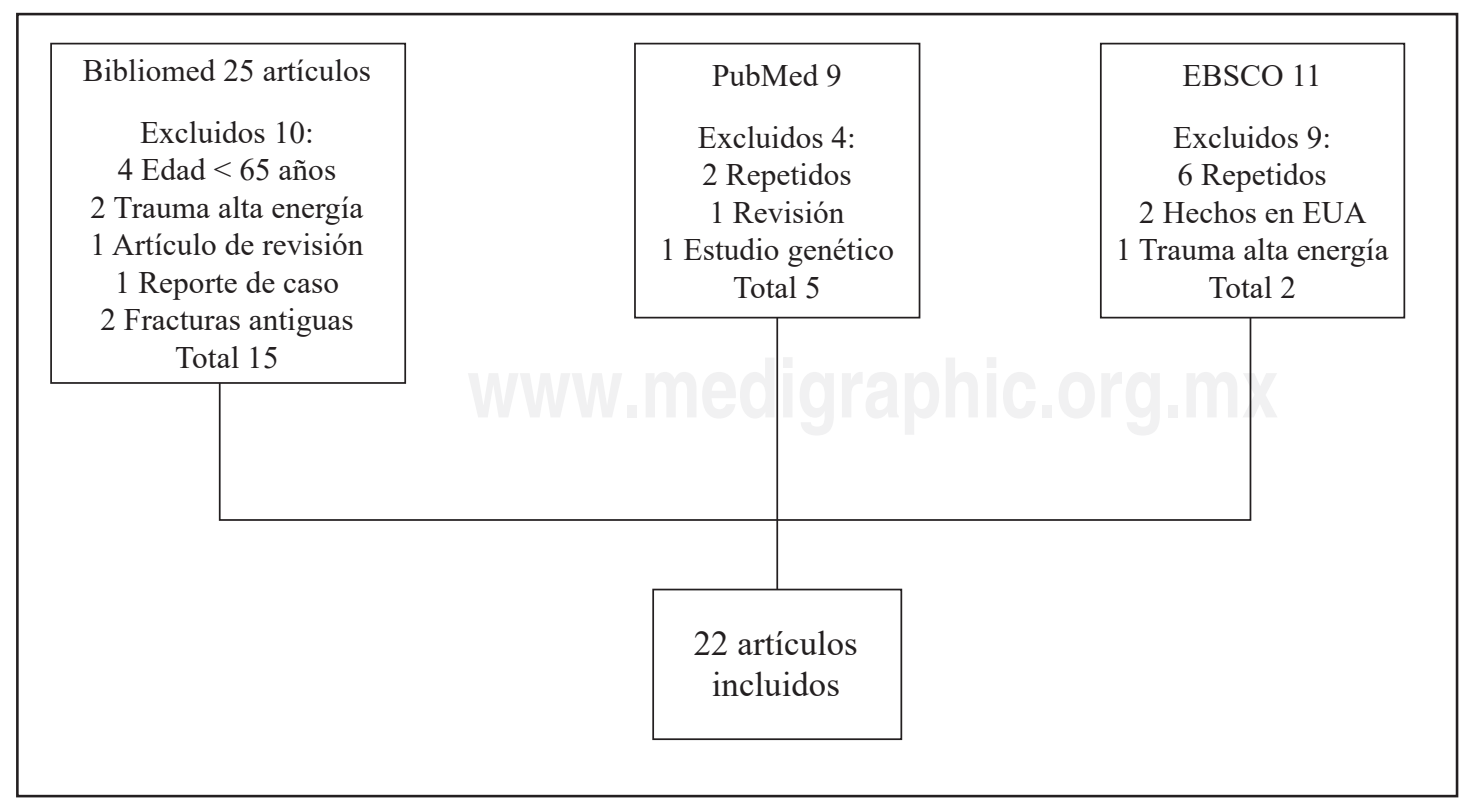

Figura 1:

Bases de datos consultadas, artículos incluidos, excluidos y motivos para ello. 


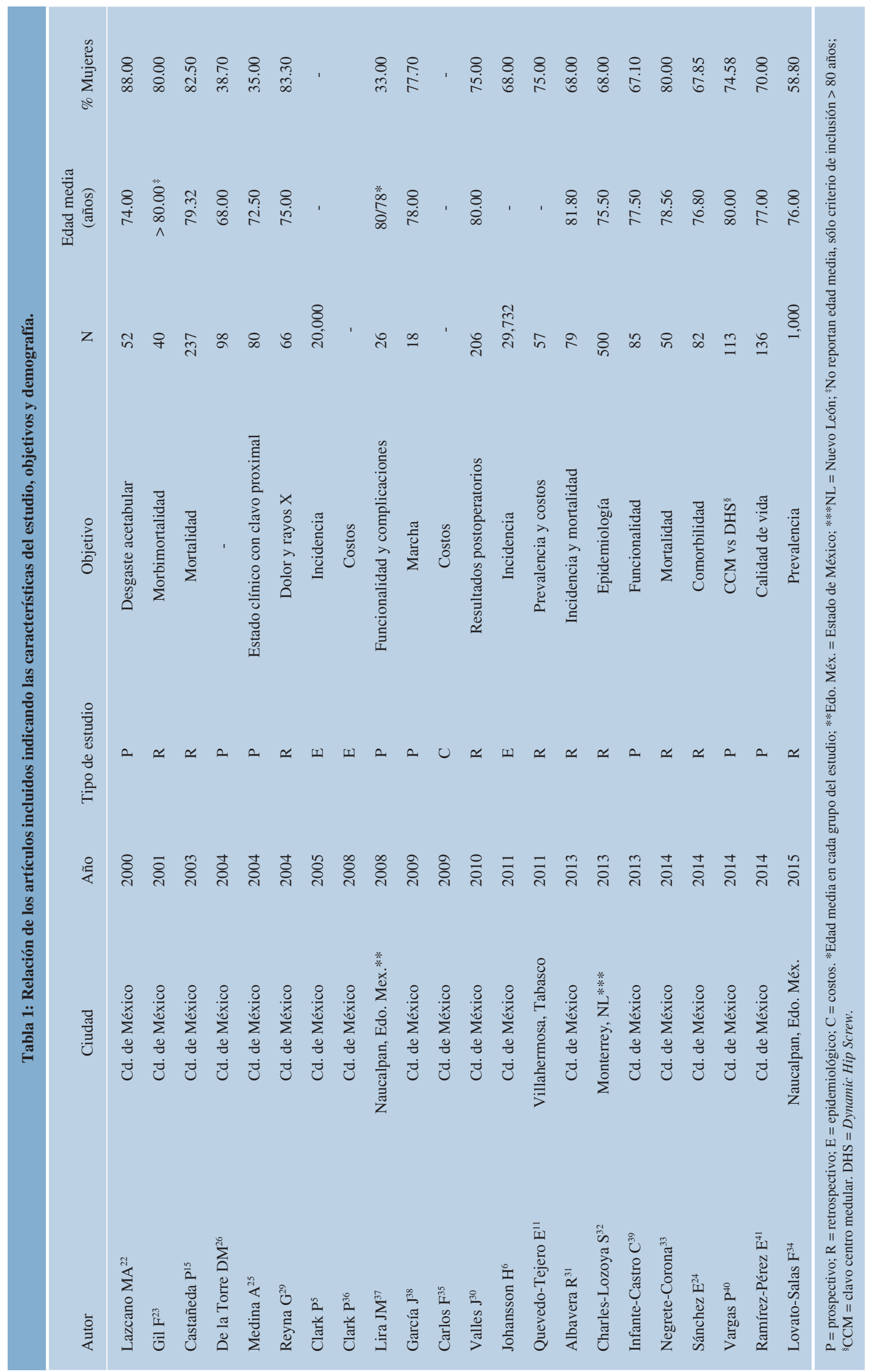


Tabla 2: Estudios sobre fractura de cadera en México que reportan comorbilidad y riesgo quirúrgico.

\begin{tabular}{|c|c|c|c|c|c|c|c|c|}
\hline Autor & HTA $(\%)$ & $\mathrm{DM}(\%)$ & Anemia (\%) & $\operatorname{ERC}(\%)$ & $\begin{array}{c}\text { Cardiopatías } \\
(\%)\end{array}$ & EPOC (\%) & $\begin{array}{c}\text { Deterioro } \\
\text { cognitivo }(\%)\end{array}$ & ASA 3-4 \\
\hline Albavera $\mathrm{R}^{31}$ & 92.0 & 64.0 & - & - & - & - & - & - \\
\hline Castañeda $\mathrm{P}^{15}$ & - & - & - & - & - & - & - & 26.7 \\
\hline Charles-Lozoya $\mathrm{S}^{32}$ & 44.6 & 32.8 & 8.4 & 11.0 & - & - & 4.8 & 44.0 \\
\hline Gil $F^{23}$ & 72.0 & 20.0 & - & 10.0 & - & 17.0 & 20.0 & 97.0 \\
\hline Infante-Castro $\mathrm{C}^{39}$ & 21.2 & 26.9 & - & - & - & - & 51.8 & - \\
\hline Lira JM $^{37}$ & 11.0 & 7.0 & 19.0 & - & - & - & - & - \\
\hline Sánchez E ${ }^{24}$ & 38.1 & 51.1 & - & 9.5 & 13.1 & 2.4 & - & - \\
\hline Valles $\mathrm{J}^{30}$ & - & 9.7 & & 0.9 & 2.4 & 6.7 & - & 18.0 \\
\hline
\end{tabular}

Tabla 3: Estudios y porcentajes de los tipos de fractura de cadera en México y el tratamiento quirúrgico instaurado.

\begin{tabular}{|c|c|c|c|c|c|c|c|c|}
\hline Registro o autor & $\begin{array}{c}\text { Intracapsular } \\
(\%)\end{array}$ & $\begin{array}{l}\text { Pertrocan- } \\
\text { térica }(\%)\end{array}$ & $\begin{array}{l}\text { Subtrocan- } \\
\text { térica }(\%)\end{array}$ & $\begin{array}{c}\text { Tx conservador } \\
(\%)\end{array}$ & $\mathrm{HAC}(\%)$ & $\operatorname{ATC}(\%)$ & Clavo CM (\%) & DHS (\%) \\
\hline Albavera $\mathrm{R}^{31}$ & 58.2 & $41.8^{*}$ & - & 32.0 & 47.0 & 10.0 & - & 43.0 \\
\hline Castañeda $\mathrm{P}^{15}$ & 56.8 & $43.2 *$ & - & 2.0 & - & - & - & - \\
\hline Charles-Lozoya $S^{32}$ & 36.0 & 56.0 & 8.0 & 0.8 & 25.6 & - & - & 25.4 \\
\hline De la Torre $\mathrm{DM}^{26}$ & - & 100.0 & - & - & 62.0 & - & - & 38.0 \\
\hline García J ${ }^{38}$ & - & 100.0 & - & - & 100.0 & - & - & - \\
\hline Gil F $\mathrm{F}^{23}$ & 52.5 & 47.5 & - & - & 67.5 & - & 20 & 12.5 \\
\hline Infante-Castro $\mathrm{C}^{39}$ & 16.5 & 83.5 & - & - & 15.3 & - & - & 84.7 \\
\hline Lazcano MA ${ }^{22}$ & 100.0 & 0 & - & - & 100.0 & - & - & - \\
\hline Lira JM ${ }^{37}$ & - & 100.0 & - & - & - & - & 50.0 & 50.0 \\
\hline Lovato-Salas $\mathrm{F}^{34 * *}$ & - & 88.8 & 12.2 & - & - & - & - & - \\
\hline Medina $\mathrm{A}^{25}$ & - & 80.0 & 20 & - & - & - & 100.0 & - \\
\hline Ramírez-Pérez E ${ }^{41}$ & - & - & - & - & $29.4^{\ddagger}$ & & $70.6^{\ddagger}$ & \\
\hline Sánchez $\mathrm{E}^{24}$ & - & 100.0 & - & - & - & - & - & - \\
\hline Valles $\mathrm{J}^{30}$ & 30.1 & 67.5 & 2.4 & - & 20.9 & - & 5.5 & 64.6 \\
\hline Vargas $\mathrm{P}^{41}$ & - & 100.0 & - & - & - & - & 31.0 & 69.0 \\
\hline
\end{tabular}

*Reúnen pertrocantéricas y subtrocantéricas en el mismo grupo (extracapsulares). **Sólo se analizaron los datos de las fracturas de cadera, aunque el trabajo incluye fracturas de fémur, meseta tibial y patela. ${ }^{\star}$ No especifican a qué porcentaje se les colocó hemiartroplastía o artroplastía total. Las fijaciones internas usaron DHS y CCM. Se reportan juntos.

$\mathrm{HAC}=$ hemiartroplastía de cadera; $\mathrm{ATC}=$ artroplastía total de cadera; $\mathrm{CCM}=$ clavo centromedular; DHS = Dynamic Hip Screw.

Tabla 4: Resultados asistenciales posteriores a la cirugía, complicaciones, tipo de marcha y mortalidad.

$\begin{array}{lcccccccc}\text { Autor } & \begin{array}{c}\text { Neumonía } \\ (\%)\end{array} & \begin{array}{c}\text { UPP } \\ (\%)\end{array} & \begin{array}{c}\text { Delirium } \\ (\%)\end{array} & \begin{array}{c}\text { Infección sitio } \\ \text { quirúrgico }(\%)\end{array} & \begin{array}{c}\text { Estancia } \\ (\text { días })\end{array} & \begin{array}{c}\text { Funcionalidad } \\ (\%)\end{array} & \begin{array}{c}\text { Marcha I/A } \\ \&(\%)\end{array} & \begin{array}{c}\text { Mortalidad } 30 \text { Mortalidad } \\ \text { días }(\%)\end{array} \\ \text { general }(\%)\end{array}$


Fractura de cadera por fragilidad en México

Tabla 5: Costos de atención por fractura de cadera en la fase aguda (ajustado a inflación).

\begin{tabular}{lccc}
\hline Autor & Año & Costo por paciente & Equivalente en dólares estadounidenses al 2018 \\
\hline Quevedo-Tejero E ${ }^{11}$ & 2011 & $5,803-11,800^{*}$ & $6,610-13,641$ \\
Carlos F55 & 2009 & 20,286 a $37,636 * *$ & $1,467.93-2,723.35$ \\
Clark P & 2008 & 3,291 a $13,777 * *$ & $1,261.75$ \\
\hline *Pesos mexicanos. **Dólares estadounidenses. & &
\end{tabular}

cols. reportan un predominio del sexo femenino de $81.5 \%{ }^{12} \mathrm{y}$ el registro estadounidense Kaiser fue de $68.6 \%{ }^{42}$

Se observó una gran variabilidad en cuanto al registro de comorbilidad. La prevalencia de hipertensión fue de 11 a $92 \%{ }^{31,37}$ y los trabajos reportaron prevalencia de diabetes que va de 20 a $30 \%,{ }^{15,23,24,30,31,32,37,39}$ esto concuerda con lo descrito por Pareja ${ }^{43}$ en España o el registro de FC de Estados Unidos. ${ }^{42}$ Sólo tres estudios identificaron la presencia de demencia con una prevalencia de 4.8 y $51.8 \%$. ${ }^{23,32,39}$ En España, en la Cohorte FONDA, ${ }^{13} 18 \%$ de pacientes tenían deterioro cognitivo. Este punto en particular se considera de especial importancia, ya que junto con la funcionalidad, el estado cognitivo previo es uno de los factores pronósticos más importantes.

En cuanto a la localización de la fractura, es difícil determinar cuál es el patrón más frecuente en México, ya que sólo un trabajo reportó los tres tipos principales de fractura de cadera, mientras que el resto de los investigadores se centraron en un solo tipo. En otros países, por ejemplo, el grupo del National Hip Fracture Database (NHFD) ${ }^{9}$ en Inglaterra encontró que las intracapsulares fueron ligeramente más frecuentes, al igual en el registro de FC de EUA; ${ }^{42}$ sin embargo, en España son un poco más frecuentes las pertrocantéreas. ${ }^{13,43}$ Los dispositivos que más se utilizan fueron el DHS, seguido de la hemiartroplastía con una tasa un poco menor que el clavo centromedular. Esta decisión compete exclusivamente al equipo quirúrgico. En lo que sí parece haber un poco de más coincidencias es en el número de pacientes que reciben una artroplastía total de cadera, que en Reino Unido fue de $7.8 \%{ }^{9}$ vs. $10 \%$ en el trabajo de Albavera. ${ }^{31}$

La complicación reportada más frecuente fue el delirium, que en el trabajo de Gil-Orbezo ${ }^{23}$ fue de $35 \%$, cifra similar a otros países como el Reino Unido con $23.6 \%{ }^{9}$ o los Estados Unidos de América con $32 \%{ }^{44}$ En cuanto a la mortalidad intrahospitalaria España, ${ }^{12}$ Reino Unido ${ }^{9}$ y EUA ${ }^{42}$ es menor de 7\%, mientras que en México los trabajos publican de 8.3 a $12.5 \% .^{23,24}$ Por otra parte, los resultados asistenciales con respecto a la movilidad muestran en el trabajo de CharlesLosoya $^{32}$ que $62 \%$ camina de manera independiente y $30 \%$ asistida, esto concuerda con lo descrito en el Reino Unido. ${ }^{9}$ El resto de los trabajos reporta buenos resultados; sin embargo, existe poca claridad en cuanto a la manera y el momento en que fue evaluada la marcha..$^{25,41}$

La mayoría de los estudios se realizaron en la Ciudad de México 5,6,15,22,24,25,26,29,30,31,33,34,35,39,40,45 y un par en el Hospital de Traumatología de Lomas Verdes del IMSS. ${ }^{34,37}$ Esta cen- tralización tan importante no nos permite conocer el comportamiento de las FC en otras partes del país y no podemos extrapolar estos resultados al resto de la República.

Llama poderosamente la atención no haber encontrado trabajos que reportaran indicadores de calidad similares a los sugeridos por el $\mathrm{BFT}^{46}$ o las guías NICE, ${ }^{20}$ como el tiempo de espera para tratamiento quirúrgico, inicio de movilización, control del dolor, utilización de bloqueos del nervio femoral. Tampoco se encontraron estudios sobre el papel de las unidades ortogeriátricas o del geriatra en resultados asistenciales o costos, esto ya ha sido reportado en otras partes del mundo con reducción en la estancia hospitalaria, costos y complicaciones en comparación con el modelo tradicional. ${ }^{13,16,17,21,43,45,47,48}$

La FC en México, como en otras partes del mundo, se presentará cada vez con mayor frecuencia y continuará afectando de manera significativa la movilidad, independencia, calidad de vida y mortalidad de los adultos mayores. Se requiere mayor difusión e investigación en México sobre la FC, los beneficios de atención multidisciplinaria, tanto en la cuestión de resultados asistenciales como en la disminución de costos. Por otra parte, no hay evidencia del cumplimiento de los indicadores de calidad. Sin embargo, la ausencia de evidencia no equivale a evidencia de ausencia. Consideramos que la mejor forma de resolver este problema es a través de la realización de estudios con metodología más estandarizada, así como de registros regionales $\mathrm{y}$, en el mejor de los casos, un Registro Nacional Mexicano de Fractura de Cadera.

No cabe duda que el objetivo final de la atención de la $\mathrm{FC}$ es reintegrar al paciente lo más pronto posible a su medio y con la mayor independencia, además de disminuir las complicaciones asociadas y todo ello con la reducción del costo de asistencia y del gasto sanitario, por lo que se debe continuar fortaleciendo los esfuerzos en la atención e investigación en la fractura de cadera.

\section{Conclusiones}

La información científica sobre FC en México es escasa, heterogénea y no permite obtener resultados concluyentes. Se requiere aumentar la cantidad y la calidad de la investigación en FC en México.

Conflicto de intereses: Los autores declaran no tener ningún conflicto de intereses. 
Bibliografía

1. Organización Mundial de la Salud (OMS). Informe mundial sobre el envejecimiento y la salud. 2015; 53. doi: 10.1017/ CBO9781107415324.004

2. Foundation IO. La carga global de la osteoporosis en cifras. 2016: 40. Disponible en: https://www.iofbonehealth.org/sites/default/files/ media/PDFs/Fact Sheets/2014-Factsheet-Burden_Osteoporosis-A4ES.pdf

3. Riera-Espinoza G. Epidemiology of osteoporosis in Latin America 2008. Salud Publica Mex. 2009; 51 Suppl 1: S52-5.

4. Gómez-García F. Morbimortalidad de fracturas de cadera en el Hospital de Traumatología Magdalena de las Salinas. IMSS. Rev Mex Ortop Traum. 1998[Acceso 19 de abril de 2018]; 2(2): 48-52. Disponible en: https://biblat.unam.mx/es/revista/revista-mexicanade-ortopedia-y-traumatologia/articulo/morbimortalidad-de-fracturasde-cadera-en-el-hospital-de-traumatologia-magdalena-de-las-salinasimss.

5. Clark P, Lavielle P, Franco-Marina F, Ramírez E, Salmerón J, Kanis $\mathrm{JA}$, et al. Incidence rates and life-time risk of hip fractures in Mexicans over 50 years of age: a population-based study. Osteoporos Int. 2005; 16(12): 2025-30.

6. Johansson H, Clark P, Carlos F, Oden A, McCloskey EV, Kanis JA. Increasing age- and sex-specific rates of hip fracture in Mexico: a survey of the Mexican Institute of Social Security. Osteoporos Int. 2011; 22(8): 2359-64

7. Azagra R, López-Expósito F, Martin-Sánchez JC, Aguyé A, Moreno $\mathrm{N}$, Cooper C, et al. Changing trends in the epidemiology of hip fracture in Spain. Osteoporos Int. 2014; 25(4): 1267-74. doi: 10.1007/ s00198-013-2586-0.

8. González-Montalvo JI, Alarcón T, Hormigo-Sánchez AI. ¿Por qué fallecen los pacientes con fractura de cadera? Med Clin (Barc). 2011; 137(8): 355-60. doi: 10.1016/j.medcli.2010.07.005.

9. Royal College of Physicians. National Hip Fracture Database (NHFD) annual report 2014. London: RCP; 2014. ISBN978-1-86016-577-1.

10. Velasco-Murillo V, Navarrete-Hernández E, Pozos-Cavanzo JL, Ojeda-Mijares RI, Camacho-Rodríguez MA. Fracturas en mujeres postmenopáusicas en el IMSS: frecuencia y costos de su atención hospitalaria. Gac Méd Méx. 2003[Acceso 19 de abril de 2018]; 139(5): 453-8. Disponible en: http://www.medigraphic.com/pdfs/gaceta/gm2003/gm035d.pdf

11. Quevedo-Tejero EC, Zavala-González MA, Hernández-Gamas AC, Hernández-Ortega HM. Hip fracture in older adults: prevalence and costs in two hospitals. Tabasco, Mexico, 2009. Rev Peru Med Exp Salud Publica. 2011; 28(3): 440-5.

12. Alarcón T, Gonzalez-Montalvo JI, Gotor P, Madero R, Otero A. A new hierarchical classification for prognosis of hip fracture after 2 years' follow-up. J Nutr Health Aging. 2011; 15(10): 919-23.

13. Sáez P, González-Montalvo JI, Alarcón-Alarcón T, Gotor-Pérez P, Martín-Maestre I. ¿Es posible mejorar la atención al paciente con fractura de cadera? Aportaciones del programa Fonda. 3a Actual en Ortogeriatría Ávila. 2015; 308.

14. Scottish Hip Fracture Audit. Scottish Hip Fracture Care Pathway Report 2017. 2017; 1-44. Disponible en: www.shfa.scot.nhs.uk.

15. Castañeda-Leeder P, Cassis-Zacarías N. Mortalidad posterior a fracturas de cadera tratadas en el Centro Médico ABC entre 1996 y 2001. An Med Asoc Med Hosp ABC. 2003; 48 (1): 33-7.

16. González-Montalvo JI, Alarcón T, Gotor P, Del Río M, Sáez P, Bárcena A. La intervención geriátrica puede mejorar el curso clínico de los ancianos frágiles con fractura de cadera. Medicina Clínica. 2001; 116(1): 1-5.

17. González-Montalvo JI, Gotor-Pérez P, Martín-Vega A, AlarcónAlarcón T, Mauleón-Álvarez de Linera JL, Gil-Garay E, et al. La unidad de ortogeriatría de agudos. Evaluación de su efecto en el curso clínico de los pacientes con fractura de cadera y estimación de su impacto económico. Rev Esp Geriatr Gerontol. 2011; 46(4): 193-9. doi: 10.1016/j.regg.2011.02.004.

18. Sáez-López P, González-Montalvo JI, Ojeda-Thies C, MoraFernández J, Muñoz-Pascual A, Cancio JM, et al. Spanish National
Hip Fracture Registry (SNHFR): a description of its objectives, methodology and implementation. Rev Esp Geriatr Gerontol. 2018; 53(4): 188-95.

19. ANZHFR Bi-National Annual Report of Hip Fracture Care 2018. Australian and New Zealand Hip Fracture Registry, August 2018. ISBN-13: 978-0-7334-3824-0.

20. National Institute of Health and Care Excellence (NICE). Hip fracture management (CG124). 2011[April 2017]: 1-19. Disponible en: https://www.nice.org.uk/guidance/cg124/resources/hip-fracturemanagement-pdf-35109449902789.

21. Sabharwal S, Wilson H. Orthogeriatrics in the management of frail older patients with a fragility fracture. Osteoporos Int. 2015; 26(10): 2387-99. doi: 10.1007/s00198-015-3166-2.

22. Lazcano-Marroquín MA, Sauri-Arce JC. Tratamiento de las fracturas subcapitales de fémur con hemiartroplastia Lazcano. An Med Asoc Med Hosp ABC. 2000; 45(4): 167-71.

23. Gil-Orbezo FI, Preciado-Aceves ME, Trueba-Davalillo C, Pino-Aznar J, Saleh-Larrañaga S. Factores pronósticos en la morbi-mortalidad en las fracturas de cadera en el anciano. Reporte de 40 casos en el Hospital Español de México. Trauma. 2001; 4(2): 52-6.

24. Sánchez-Hernández E, Mejía-Rohenes LC, Benítez-Romero A. Comorbilidades en pacientes operados por fractura transtrocantérica. Rev Esp Med Quir. 2014; 19(2): 145-51.

25. Medina-Castellanos A, Reséndiz A, Pozos E, Gómez T, Guerrero N, Almanza A, et al. Tratamiento con clavo proximal femoral en fracturas trans y transubtrocantéricas. Acta Ortop Mex. 2004; 18(6): 231-4.

26. de la Torre-González DM, Góngora-López J, Galeana-López JC. Tratamiento quirúrgico de las fracturas intertrocantéricas de la cadera en el anciano. Trauma. 2004; 7(2): 53-8.

27. Royal College of Physicians. Best Practice Tariff (BPT) for fragility hip fracture care user guide. 2010. Disponible en: http://nhfd.co.uk

28. Fxtop.com/es/convertidor-divisas-pasado.php / fxtop.com/es/ convertidor-divisas-pasado.php

29. Reyna-Olivera G, Harb-Peña EJ. Comparación clínico-radiográfica del fresado o no fresado acetabular en la hemiartroplastía de cadera. Acta Ortop Mex. 2004; 18(3): 96-9.

30. Valles-Figueroa JFJ, Malacara-Becerra M, Gómez-Mont-Landerreche G, Suárez-Ahedo CE, Cárdenas-Elizondo JL. Tratamiento quirúrgico de las fracturas de cadera. Acta Ortop Mex. 2010; 24(4): 242-7. Disponible en: http://www.medigraphic.com/pdfs/ortope/or-2010/ or104f.pdf.

31. Albavera-Gutiérrez RR, López-García R, Antonio-Romero CE, Gurrola-Mendoza K, Montero-Quijano MG, Pérez-Solares A, et al. Mortalidad de pacientes con fractura de cadera a cinco años de evolución en el Hospital Regional General Ignacio Zaragoza. Rev Esp Méd Quir. 2013; 18(1): 31-6.

32. Charles-Lozoya S, Treviño-Pérez J, Rangel-Flores JM. Aspectos clínico-epidemiológicos y terapéuticos en los pacientes con fractura de cadera. Acta Ortop Mex. 2013; 27(6): 375-9.

33. Negrete-Corona J, Alvarado-Soriano JC, Reyes-Santiago LA. Fractura de cadera como factor de riesgo en la mortalidad en pacientes mayores de 65 años. Estudio de casos y controles. Acta Ortop Mex. 2014; 28(6): 352-62. Disponible en: http://www.medigraphic.com/pdfs/ ortope/or-2014/or146c.pdf

34. Lovato-Salas F, Luna-Pizarro D, Oliva-Ramírez SA, Flores-Lujano J, Núñez-Enríquez JC. Prevalencia de fracturas de cadera, fémur y rodilla en la Unidad Médica de Alta Especialidad Hospital de Traumatología y Ortopedia "Lomas Verdes" del Instituto Mexicano del Seguro Social. Acta Ortop Mex. 2015; 29(1): 13-20. Disponible en: http://pesquisa.bvsalud.org/portal/resource/pt/mdl-26999921.

35. Carlos F, Clark P, Maciel H, Tamayo JA. Direct costs of osteoporosis and hip fracture: an analysis for the Mexican Social Insurance Health Care System. Salud Publica Mex. 2009; 51 Suppl 1: S108-13. doi: 10.1590/S0036-36342009000700014.

36. Clark P, Carlos F, Barrera C, Guzman J, Maetzel A, Lavielle P, et al. Direct costs of osteoporosis and hip fracture: an analysis for the Mexican healthcare system. Osteoporos Int. 2008; 19(3): 269-76. doi: 10.1007/s00198-007-0496-8.

37. Lira-Romero JM, Arenas-Díaz R, Montero-Oropeza Y. Fracturas pertrocantéricas en adultos mayores tratados mediante el tornillo 
dinámico de cadera vs placa de compresión percutánea. Acta Ortop Mex. 2008; 22(2): 115-9.

38. García-Juárez JD, Tohen-Bienvenu A, García-Hernández A, DávilaSheldon O. Endoprótesis femoral cementada con reemplazo de calcar en fracturas intertrocantéricas de cadera en el viejo. Acta Ortop Mex. 2009; 23(6): 326-30. Disponible en: http://www.medigraphic.com/ pdfs/ortope/or-2009/or096b.pdf

39. Infante-Castro CI, Rojano-Mejía D, Ayala-Vázquez G, AguilarEsparza G. Factores pronósticos de funcionalidad en adultos mayores con fractura de cadera. Cir Cir. 2013; 81(2): 125-30.

40. Vargas-Ortiz PD, Antonio-Romero CE, Mejía-Rohenes LC. Ventajas funcionales y biomecánicas del clavo centromedular vs. el tornillo dinámico de cadera en fracturas intertrocantéricas. Rev Esp Méd Quir. 2014; 19: 156-62. Disponible en: http://www.medigraphic.com/pdfs/ quirurgicas/rmq-2014/rmq142d.pdf

41. Ramírez-Pérez E, Clark P, Carlos F, Camacho A, Franco-Marina F. Health-related quality of life after surgery for hip fracture: a multicentric study in Mexican population. Medwave. 2014[Accessed March 16, 2018]; 14(5): e5972. Disponible en: http://www.ncbi.nlm. nih.gov/pubmed/25211398.

42. Inacio MC, Weiss JM, Miric A, Hunt JJ, Zohman GL, Paxton EW A community-based hip fracture registry: population, methods, and outcomes. Perm J. 2015; 19(3): 29-36. doi: 10.7812/TPP/14231 .
43. Pareja-Sierra T, Rodríguez-Solisa J, Alonso-Fernández P, TorralbaGonzález de Suso M, Hornillos-Calvo M. Intervención geriátrica en el anciano ingresado por fractura de cadera en el Hospital Universitario de Guadalajara: repercusión clínica, asistencial y económica. Rev Esp Geriatr Gerontol. 2017; 52(1): 27-30. doi: 10.1016/j. regg.2016.02.001.

44. Marcantonio ER, Flacker JM, Wright RJ, Resnick NM. Reducing delirium after hip fracture: a randomized trial. J Am Geriatr Soc. 2001[Accessed April 30, 2018]; 49(5): 516-22. Disponible en: http:// www.ncbi.nlm.nih.gov/pubmed/11380742.

45. González-Montalvo JI, Alarcón T, Mauleón JL, Gil-Garay E, Gotor P, Martín-Vega A. The orthogeriatric unit for acute patients: a new model of care that improves efficiency in the management of patients with hip fracture. Hip Int. 2010; 20(2): 229-35. Disponible en: http://onlinelibrary. wiley.com/o/cochrane/clcentral/articles/007/CN-00761007/frame.html.

46. Khan SK, Weusten A, Bonczek S, Tate A, Port A. The best practice tariff helps improve management of neck of femur fractures: a completed audit loop. Br J Hosp Med (Lond). 2013; 74(11): 644-7.

47. Castanheira L, Palmeiro A, Fresco P, Macedo AF. A medicação crónica no período perioperatório. Perfil de utilização e gestão de risco. Acta Med Port. 2011; 24(6): 893-8.

48. Briggs RS. Orthogeriatric care and its effect on outcome. J R Soc Med. 1993; 86(10): 560-2. 